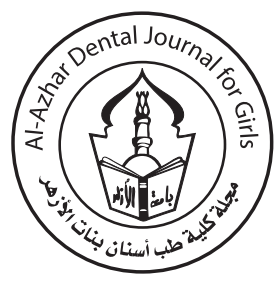

\title{
Effect of Oral Rinses on Color Change Behaviour of Three Types of Provisional Fixed Partial Denture Materials
}

\author{
Khaled Haggag( $^{(1)}$, Muhammad Abbas ${ }^{(2)}$ and Zainab EL Sharkawy ${ }^{(3)}$
}

Codex : 13/1801

azhardentj@azhar.edu.eg

http://adjg.journals.ekb.eg

\section{KEYWORDS}

Oral Rinses,

Color Change,

Fixed Partial Denture

\begin{abstract}
Objectives: The present study aimed to comparatively evaluate the color variations of three different commercially available provisional restorative materials, using computerized spectrophotometry before and after immersion in artificial saliva and three other different mouth rinses Materials and Methods: One hundred and twenty samples (40 of each material; Revotek LC, TempSpan, Jet tooth shade) were prepared in the form of discs (10 $\mathrm{mm}$ diameter and $2 \mathrm{~mm}$ thickness), and these samples were randomly divided into 4 subgroups for the solutions; chlorohexidine, Tantum V and Oracin mouth washes and artificial saliva( $(n=10)$. The discoloration solutions were changed twice a day throughout the three weeks. The color values $\left(\mathrm{L}^{*}, \mathrm{a}^{*}, \mathrm{~b}^{*}\right)$ of each specimen were measured before and after exposure with a Reflective spectrophotometer. Results: regardless to staining solutions, it was shown that TempSpan material group recorded the highest color change mean value $(3.68 \pm 0.39 \Delta \mathrm{E})$ followed by Revotek LC material group mean value $(1.56 \pm 0.27 \Delta \mathrm{E})$ while Jet tooth shade material group recorded the lowest color change mean value $(1.4 \pm 0.46 \Delta \mathrm{E})$.on the other hand, A. saliva recorded the highest color change mean value $(2.78 \pm 1.2 \Delta \mathrm{E})$ followed by Tantum $\mathrm{V}$ solution mean value $(1.72 \pm 0.31 \Delta \mathrm{E})$ then Oracin solution mean value $(1.5 \pm 0.4 \Delta \mathrm{E})$ while CHX staining solution recorded the lowest color change mean value $(1.32 \pm 0.4$ $\Delta \mathrm{E})$. Conclusions:1- All the bis-acryl resins and PMMA-based provisional materials showed clinically perceptible color changes after exposure to oral rinses. 2- Among the three provisional restorative materials, Tempspan was found to be significantly the least color stable material and the least stain was taken up by Jet. 3- There were no significant differences between the discoloration effects of Tantum V, Oracin and Chlorohexidine mouth washes on the three tested provisional materials.
\end{abstract}

1. Assistant Professor, Crowns and Bridges Department, Faculty of Dental Medicine, Al Azhar University, Egypt

2. Assistant Professor, Dental Biomaterials Department, Faculty of Dental medicine, Al-Azhar University, Egypt

3. Lecturer, Crowns and Bridges Department, Faculty of Dental Medicine for Girls, Al Azhar University, Egypt 


\section{INTRODUCTION}

Provisional crowns and fixed partial dentures (FPDs) are a considerable part of prosthodontic treatment, ${ }^{1}$ that denotes serving for a period of time as a necessary step in providing for the final restorations.

The provisional helps to maintain periodontal health, maxillo-mandibular relationships, and continued evaluation of fixed prosthodontic treatment plans. ${ }^{2}$ It should also provide a protective shield for the dental tissues against thermal and chemical insults, rehabilitate the normal function and finally it should be esthetically pleasing to the patient. ${ }^{3}$

In practice, provisional restorations could be used as a short:7-10 days, medium:6 weeks or a long term: 6 months provisional, ${ }^{4}$ such as cases which need extensive prosthodontic treatment for full mouth rehabilitations, tooth wear, and in partially edentulous patients undergoing implant therapy. ${ }^{5}$

As a demand these restorations must not only provide an initial shade match, but also maintain smooth, highly polished surface \& color stability over a period of time, particularly when it is in the esthetic zone. ${ }^{6}$

Unfortunately, provisional restoration could still experience a visible color change due to many factors including type of material, patient's diet, oral hygiene, water sorption as well as incomplete polymerization. To limit potential discoloration process, some manufacturers have certain recommendations for their products regarding polishing, others provide a range of materials with continuous ongoing improvements to meet the increasing needs of the patient $\&$ avoid additional expense for replacement. ${ }^{7}$

Despite some limitations, the quantitative evaluation of color difference $(\Delta \mathrm{E})$ with a spectrophotometer grants many advantages like repeatability, sensitivity, objectivity. ${ }^{8}$ If a material is completely color stable, no color difference will be detected by its exposure to the testing apparatus.
Materials used for fabricating provisional restorations include auto polymerizing polymethyl methacrylate, polyethylene methacrylate, polyvinyl methacrylate, urethane methacrylate, bis-acryl and even micro filled resin; that can be either chemically or light polymerized or both. ${ }^{9}$

It is still a contentious issue in research as to which type of material has the better color stability. ${ }^{10-}$ Some researchers have concluded that most of provisional restorative materials have only acceptable color stability for a short period of time and that they all discolor over a period of time if exposed to staining solutions. ${ }^{13,14}$

Various studies have been reported on the influence of staining materials like tea, coffee, red wine on the provisional materials. ${ }^{11,15,16}$ An evaluation of the color stability of four provisional restorative materials after 48 hours of immersion in a staining solution was performed. It was found that, the methyl methacrylate-based provisional restorative material more color stable than the auto polymerized and light- polymerized composite provisional materials tested. ${ }^{9}$

Color stability of provisional materials immersed in various staining solutions for 1 month was determined. After this period the methyl methacrylate materials exhibited the best color stability and bis-acryl the worst. ${ }^{11}$

Oral rinses are widely used during this interim period, because of their anti-inflammatory, antiseptic, and analgesic properties. ${ }^{11,17}$ They can also reduce plaque levels and improve gingival health. ${ }^{18}$ It was reported that the use of oral rinses for three weeks caused discoloration of natural teeth which was clinically unacceptable.

There is still a lack of literature evidence regarding the effect of these mouth rinses on the color stability of provisional restorations. Hence, this in vitro study aimed to comparatively evaluate the color variations of three different commercially available provisional restorative materials, using computerized spectrophotometry before and after immersion in artificial saliva and three different mouth rinses. 


\section{MATERIALS AND METHODS}

Three different provisional materials (Revotek LC-GC Corp, TempSpan Pentron Clinical Technologies and Jet self-cure tooth shade powder), three staining solutions (chlorhexidine, benzydamine $\mathrm{HCl}$, alcohol-free mouthwash) and artificial saliva as control were used in this study.

\section{Preparation of samples}

One hundred and twenty samples, (40) of each material were prepared in the form of discs $(10 \mathrm{~mm}$ diameter and $2 \mathrm{~mm}$ thickness) using especially designed custom made split brass mold (fig.1) with two mold cavities, and these samples were randomly divided into 4 subgroups for the solutions $(n=10)$

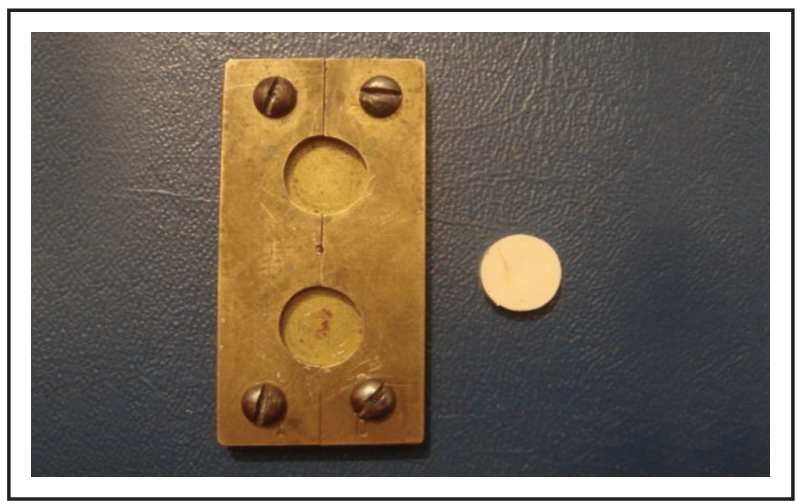

Fig. (1) Split circular brass mold used to create disc samples
The product names, types of materials and manufacturers are listed in Table 1.

The samples were made with the three different provisional materials (Revotek LC, TempSpan and Jet tooth shade) as mentioned above to compare the color stability.

Revotek LC (GC Corporation, Japan); Lightcured single component composite resin. Group 1 - forty samples made, ten samples each for oral rinse solution subgroup. the mold was filled with the material using the spatula provided. and covered by myler strip over which glass plate was pressed. A light emitting diode (LED) powered visible lightcuring unit (Spectrum $800^{\mathrm{TM}}$ curing unit; Dentsply Caulk, USA) was used for 40 s in fast-cure mode $(440-480 \mathrm{~nm})$

TempSpan (Pentron Clinical Technologies, LLC); Dual-cure resin system. Group 2 - forty samples made, ten samples each for oral rinse solution subgroup. The material was mixed using the amount of each component that was delivered by three turns of the dispensing syringes. The material was dispensed into the mold and allowed to autopolymerize. To complete curing, light cure for 20 seconds. To remove oxygen inhibited layer each disc was treated with $99.9 \%$ ethanol.

Table (1) Materials names, types and manufacturers used in the study

\begin{tabular}{|c|c|c|c|}
\hline Brand & Material type & Manufacturer & Lot \# \\
\hline Revotek $\boldsymbol{L C}$ & light-cure urethane dimethacrylate resin & GC Dental Products, Tokyo, Japan & 1510291 \\
\hline TempSpan & $\begin{array}{c}\text { Dual-cure Bis-GMA composite resin } \\
\text { system }\end{array}$ & $\begin{array}{c}\text { Pentron Clinical Technologies, LLC, } \\
\text { USA }\end{array}$ & 6006379 \\
\hline Jet tooth shade & Chemical cure acrylic resin powder and \\
liquid & Lang Dental Manufacturer, USA & $\begin{array}{c}\text { P;2023215 } \\
\text { L; } 144215 \mathrm{BW}\end{array}$ \\
\hline Hexitol & Chlorhexidine HCL 125mg/100 ml & Arab drug company, Cairo, Egypt & 530330 \\
\hline Tantum Verde & Benzydamine HCL 0.15 g & EIPICO, under license of ACR & 1510794 \\
\hline Oracin & $\% 0.064$ thymol & Angelini, Italy & 150125 \\
\hline
\end{tabular}


Jet tooth shade (Lang Dental Manufacturer, USA); Chemically cured two component systems. Group 3 - forty samples made, ten samples each for oral rinse solution subgroup. The materials were dispensed, manipulated, and polymerized according to the manufacturers' instructions. The chemically cured materials were mixed in a mixing cup according to the manufacturers' suggested ratio, using a glass spatula until a homogeneous mix was obtained according to the manufacturer's directions. The materials were then placed separately into the mold as mentioned before and allowed to auto-polymerize.

After completely setting, the excess provisional materials were ground by hand lapping with a 1000grit silicon paper for 10 seconds. The surfaces of the samples were polished by one operator for $15 \mathrm{sec}$ onds using pumice, which was followed by rinsing with distilled water to remove any debris before immersion. All the samples were kept dry at room temperature until the rest of samples were fabricated.

\section{Preparation of the Staining Solutions}

Artificial saliva was prepared, and it consisted of the following ingredients: $1 \mathrm{~g}$ sodium carboxymethylcellulose, $4.3 \mathrm{~g}$ xylitol, $0.1 \mathrm{~g}$ potassium chloride, 5 $\mathrm{mg}$ calcium chloride, $40 \mathrm{mg}$ potassium phosphate, 1 $\mathrm{mg}$ potassium thiocyanate and $100 \mathrm{~g}$ deionized water. ${ }^{19}$ Each oral rinse (chlorhexidine, benzydamine $\mathrm{HCl}$, alcohol-free mouthwash) and the artificial saliva were maintained in a dark environment at $37^{\circ} \mathrm{C}$ $\pm 1^{\circ} \mathrm{C}$ to stimulate the conditions in oral cavity. The discoloration solutions were changed twice a day throughout the three weeks.

\section{Evaluating color stability}

All the samples were stored in artificial saliva at $37^{\circ} \mathrm{C} \pm 1^{\circ} \mathrm{C}$ for 24 hours. The rehydration simulated the first day of service for provisional restorations in the oral environment. The color values $\left(\mathrm{L}^{*}, \mathrm{a}^{*}, \mathrm{~b}^{*}\right)$ of each specimen were measured before exposure with a Reflective spectrophotometer (X-Rite, model RM200QC, Neu-Isenburg, Germany). The aperture size was set to $4 \mathrm{~mm}$ and the samples were exactly aligned with the device. A white background was selected, and measurements were made according to the CIE $\mathrm{L}^{*} \mathrm{a} * \mathrm{~b} *$ color space relative to the CIE standard illuminant D65. The CIE L*a*b* system is an approximately uniform color space with coordinates for lightness, namely white-black $\left(\mathrm{L}^{*}\right)$, redness-green $\left(a^{*}\right)$, and yellowness-blueness $\left(b^{*}\right)$. The measurements were repeated 3 times for each specimen, and the mean values of $\mathrm{L}^{*}, \mathrm{a}^{*}, \mathrm{~b}^{*}$ were calculated. After the baseline color measurements were obtained, the samples were immersed into the discoloration solutions. After 2 minutes of immersion in the oral rinses, the samples were immersed in artificial saliva. Thus, the samples were exposed to oral rinses for a total of 84minutes, which is equivalent to the time of mouth rinse use for 3 weeks. ${ }^{20}$ The remaining samples from each material served as a control group and were stored only in the artificial saliva during the 3 weeks. After three weeks of immersion, the samples were rinsed with distilled water for 5 minutes and gently brushed with a soft toothbrush for 15 seconds. At this point, color measurements were recorded with the same spectrophotometer, and these measurements were performed under the same conditions and in the same manner described for the baseline measurements. The calculation of the color variation $\Delta \mathrm{E}^{*}$ between two color positions (three weeks of storage and baseline) in 3-dimensional $\mathrm{L}^{*} \mathrm{a} * \mathrm{~b}^{*}$ color space is as follows:

$$
\Delta \mathrm{E}_{\mathrm{CIELAB}}=\left(\Delta \mathrm{L}^{* 2}+\Delta \mathrm{a}^{* 2}+\Delta \mathrm{b}^{* 2}\right)^{1 / 2}
$$

Where: $\mathrm{L}=*$ lightness $(0-100), \mathrm{a}=*$ change the color of the axis (red/green) and $b=*$ color variation axis (yellow/blue).

\section{Statistical analysis}

Data analysis was performed in several steps. Initially, descriptive statistics for each group results. Two-way ANOVA was done to detect effect of each variable (materials groups and oral rinse staining solutions subgroups). One-way ANOVA followed by pair-wise Tukey's post-hoc tests were performed to detect significance between subgroups and main group. Statistical analysis was performed using Asistat 7.6 statistics software for Windows 
(Campina Grande, Paraiba state, Brazil). P values $\leq 0.05$ are considered to be statistically significant in all tests.

\section{RESULTS}

\section{Color change $(\Delta E)$}

Color change $(\Delta \mathrm{E})$ results) Mean $\pm \mathrm{SD})$ for all groups as function of oral rinse staining solutions are summarized in table (2) and figure (2).

Effect of material; regardless to staining solutions totally it was shown that TempSpan material group recorded the highest color change mean value $(3.68 \pm 0.39 \Delta \mathrm{E})$ followed by Revotek $\boldsymbol{L C}$ material group mean value $(1.56 \pm 0.27 \Delta \mathrm{E})$ while Jet tooth shade material group recorded the lowest color change mean value $(1.4 \pm 0.46 \Delta \mathrm{E})$. The difference between materials groups means values was statistically significant as indicated by two-way ANOVA $(\mathrm{F}=32.53, \quad \mathrm{P}=<0.0001<0.05)$. Pair-wise Tukey's post-hoc test showed non-significant $(\mathrm{p}>0.05)$ difference between Revotek $L C$ and Jet tooth shade groups

Effect of oral rinse staining solution; irrespective of materials groups totally it was shown that A. saliva recorded the highest color change mean value $(2.78 \pm 1.2 \Delta \mathrm{E})$ followed by Tantum $\boldsymbol{V}$ solution mean value $(1.72 \pm 0.31 \Delta \mathrm{E})$ then Oracin solution mean value $(1.5 \pm 0.4 \Delta \mathrm{E})$ while $\boldsymbol{C H} \boldsymbol{X}$ staining solution recorded the lowest color change mean value $(1.32 \pm 0.4 \Delta \mathrm{E})$. The difference between $\boldsymbol{A}$. saliva and staining solutions means values was statistically significant as indicated by two-way ANOVA $(\mathrm{P}=<0.0001<0.05)$. Pair-wise Tukey's post-hoc test showed non-significant $(\mathrm{p}>0.05)$ difference between $\boldsymbol{C H} \boldsymbol{X}$, Tantum $\boldsymbol{V}$ and Oracin solutions

\section{Interaction between variables}

With A. saliva solution; it was found materials color mean values influenced significantly where (TempSpan > Jet tooth shade >Revotek LC) as demonstrated by one-way ANOVA ( $\mathrm{F}=28.5$, $\mathrm{P}=<0.0001<0.05)$. Pair-wise Tukey's post-hoc test showed non-significant $(\mathrm{p}>0.05)$ difference between Revotek LC and Jet tooth shade groups
With $\boldsymbol{C H} \boldsymbol{X}$ solution; also, it was found materials color mean values influenced significantly where (Revotek LC > TempSpan > Jet tooth shade) as verified by one way ANOVA ( $\mathrm{F}=90.3$, $\mathrm{P}=<0.0001<0.05)$.

With Tantum $\boldsymbol{V}$ solution; materials color significantly affected where (Jet tooth shade> TempSpan $>$ Revotek $\boldsymbol{L C}$ ) as revealed by one way ANOVA $(\mathrm{F}=109.7, \mathrm{P}=<0.0001<0.05)$.

With Oracin solution; materials color significantly affected where (TempSpan > Revotek LC > Jet tooth shade) as revealed by one way ANOVA $(\mathrm{F}=3.9, \mathrm{P}=0.04<0.05)$. Pair-wise Tukey's post-hoc test showed non-significant $(\mathrm{p}>0.05)$ difference between (Revotek LC and Jet tooth shade) and (Revotek LC and TempSpan) groups

In Revotek $\mathbf{L C}$ materials group; oral rinse solutions influenced the color stability significantly where ( $\boldsymbol{C H} X \geq A$. saliva $\geq$ Oracin $\geq$ Tantum $\boldsymbol{V}$ ) as revealed by one way ANOVA $(\mathrm{F}=4.7, \mathrm{P}=0.0121<0.05)$. Pairwise Tukey's post-hoc test showed non-significant ( $>>0.05$ ) difference between A. saliva, Tantum $\boldsymbol{V}$ and Oracin solutions. Also non-significant ( $\mathrm{p}>0.05)$ difference found between A. saliva, $\boldsymbol{C H} \boldsymbol{X}$ and Oracin solutions.

In TempSpan materials group; oral rinse solutions affected on the color stability significantly where ( $\boldsymbol{A}$. saliva $\geq$ Oracin $\geq$ Tantum $\boldsymbol{V} \geq \boldsymbol{C H} \boldsymbol{X})$ as revealed by one way ANOVA $(\mathrm{F}=38.9, \mathrm{P}=<0.0001<0.05)$. Pairwise Tukey's post-hoc test showed non-significant ( $>>0.05$ ) difference between Tantum $\boldsymbol{V}$ and Oracin solutions. Also non-significant ( $\mathrm{p}>0.05)$ difference found between $\boldsymbol{C H} \boldsymbol{X}$ and Tantum $\boldsymbol{V}$ solutions.

In Jet tooth shade materials group; oral rinse solutions affected on the color stability significantly where (Tantum $V \geq A$. saliva $\geq$ Oracin $\geq$ $\boldsymbol{C H} \boldsymbol{X})$ as revealed by one way ANOVA $(\mathrm{F}=7.2$, $\mathrm{P}=0.0019<0.05)$. Pair-wise Tukey's post-hoc test showed non-significant $(\mathrm{p}>0.05)$ difference between Tantum $\boldsymbol{V}$ and $\boldsymbol{A}$. saliva solutions. Also nonsignificant ( $\mathrm{p}>0.05$ )difference found between $\boldsymbol{C H} \boldsymbol{X}$ and Oracin solutions. 
Table (2) Color change $(\Delta E)$ results $(M e a n \pm S D)$ for all groups as function of oral rinse staining solutions

\begin{tabular}{|c|c|c|c|c|c|c|c|c|c|c|c|c|}
\hline \multirow{2}{*}{ Variables } & \multicolumn{4}{|c|}{ Revotek LC } & \multicolumn{4}{|c|}{ TempSpan } & \multicolumn{4}{|c|}{ Jet tooth shade } \\
\hline & $\Delta \mathrm{L}$ & $\Delta \mathrm{a}$ & $\Delta b$ & $\Delta \mathrm{E}$ & $\Delta L$ & $\Delta \mathrm{a}$ & $\Delta b$ & $\Delta \mathrm{E}$ & $\Delta \mathrm{L}$ & $\Delta \mathrm{a}$ & $\Delta b$ & $\Delta \mathrm{E}$ \\
\hline A. saliva & -0.1 & -0.4 & -1.2 & $1.62^{\mathrm{B}}{ }_{\mathrm{ab}} \pm 0.48$ & -1.07 & -2.7 & -3.43 & $4.72^{\mathrm{A}} \pm 0.05$ & -0.77 & 1 & 0.57 & $2^{\mathrm{B}} \pm 1.34$ \\
\hline CHX & -0.3 & 0.63 & -1.6 & $1.88^{\mathrm{A}} \pm 0.16$ & -0.23 & 1.3 & -0.17 & $1.36^{\mathrm{B}}{ }_{\mathrm{c}} \pm 0.12$ & -0.33 & -0.37 & 0.43 & $0.7^{\mathrm{C}}{ }_{\mathrm{b}} \pm 0.19$ \\
\hline Tantum V & -1.1 & -0.5 & -0.2 & $1.26^{\mathrm{C}}{ }_{\mathrm{b}} \pm 0.13$ & -0.37 & 1.63 & -0.4 & $1.83^{\mathrm{B}}{ }_{\mathrm{bc}} \pm 0.1$ & -1.43 & -0.23 & -1.4 & $2.1^{\mathrm{A}}{ }_{\mathrm{a}} \pm 0.06$ \\
\hline Oracin & -1.2 & -0.1 & -0.1 & $1.46_{\mathrm{ab}}^{\mathrm{AB}} \pm 0.33$ & 0.61 & 0.64 & -0.66 & $2.1_{\mathrm{b}}^{\mathrm{A}} \pm 1.24$ & -0.27 & -0.23 & 0.07 & $0.9^{\mathrm{B}}{ }_{\mathrm{b}} \pm 0.28$ \\
\hline Total & -0.7 & -0.1 & -0.8 & $1.56 \pm^{\mathrm{B}} 0.27$ & -0.53 & -0.5 & -2.02 & $3.68^{\mathrm{A}} \pm 0.39$ & -0.7 & 0.04 & -0.1 & $1.4^{\mathrm{B}} \pm 0.46$ \\
\hline$P$ value & \multicolumn{4}{|c|}{$0.0121 *$} & \multicolumn{4}{|c|}{$<0.0001 *$} & \multicolumn{4}{|c|}{$0.0019^{*}$} \\
\hline
\end{tabular}

Different superscript large letter in same row indicating significant between materials (Tukey's $p<0.05$ )

Different subscript small letter in same column indicating significant between solutions (Tukey's $p<0.05$ )

*; significant $(p<0.05) \quad n s ;$ non-significant $(p>0.05)$

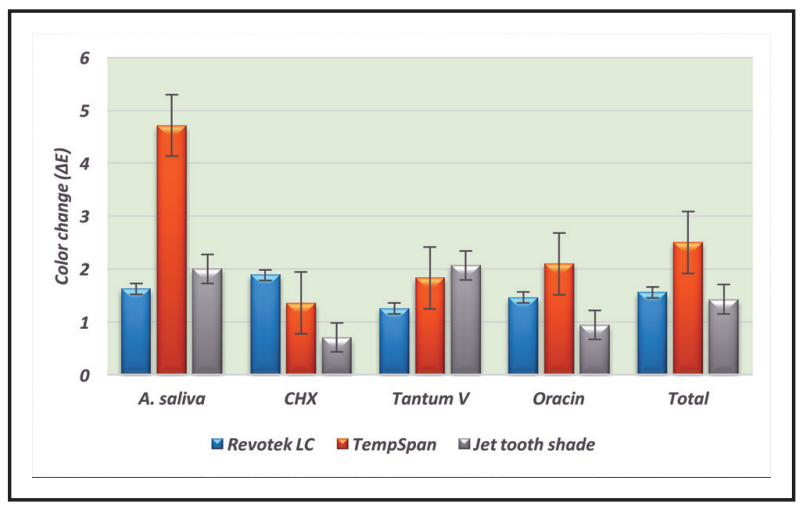

Fig. (2) Column chart of the mean values of color change all groups as function of oral rinse staining solutions

\section{DISCUSSION}

Provisional crown and fixed partial denture restorations serve many purposes in prosthodontics, including restoration of function, protection of the dental and periodontal tissues, stabilization of the occlusion..$^{21,22}$

They can also act as diagnostic aids before fabrication of the final restoration regarding aesthetics and allow for the resolution of gingival inflammation and periapical lesions. ${ }^{22,23}$

All these purposes are important but, aesthetics of the provisional restoration is usually of prime importance to the patient especially with long term provisional or when used in the aesthetic zone..$^{21-24}$

Thermoplastic acrylic [PMMA and methyl methacrylate (MMA)] materials have been traditionally used as the provisional material of choice and have, to a certain extent, met many of these mechanical and physical requirements. However, the more modern bis-acryl composite temporization materials, have become an increasingly popular choice, due in part to their improved mechanical properties, lower setting temperature, reduced polymerization shrinkage, good polishability as well as easy handling..$^{25-27}$

Color changes may occur over time when these provisional restorations contact pigmented solutions such as coffee or tea. ${ }^{21,24}$ Most provisional materials are subject to sorption, a process of absorption and adsorption of liquids that occurs relative to environmental conditions. ${ }^{6,10}$ The degree of staining is also affected by the concentration of the staining agents and the amount of time the materials are exposed to the staining agents. ${ }^{24}$ Discoloration by some materials such as tea is due to adsorption of the polar colorants onto the surface of the restorative materials and this can be removed by tooth brushing, whereas discoloration by other staining solutions such as coffee is due to both adsorption and absorption of the colorants into the restorative material. ${ }^{28,29}$ 
It was reported that incomplete polymerization \& chemical reactivity considered as factors that affect color stability of these restorations. ${ }^{12,24}$ Porosity and surface quality of provisional restorations, ${ }^{10,30}$ can also influence color changes

Occasional administration of oral rinses after tooth preparation to promote periodontal health, whereas provisional restorations assist in the maintenance of periodontal health and promote guided tissue healing by providing a matrix for the surrounding gingival tissues. ${ }^{18}$ They can affect the color stability of provisional restorations and cause discolorations secondary to dietary factors and medications.

The assessment of color can be visual which is subjective, physiologic process that tends to vary from person to another due to many factors like illuminant position, object being observed, color characteristics of the illuminant, fatigue, aging, metamerism, and also the environment state. ${ }^{31,32}$ Use of a spectrophotometer as a standardized, reliable tool would potentially eliminate such errors. ${ }^{33}$

In the present study shade changes were analyzed using the CIE $\mathrm{L} * \mathrm{a} * \mathrm{~b} *$ color system. It allows a summary value, $\Delta \mathrm{E}$, to be calculated and used as a color matching tolerance. ${ }^{34}$ In this system, L* is defined as the value, of the specific color, whereas $a *$ and $b *$ specify the actual hue. Positive a* values indicate a predominance of red hue, while negative values indicate a predominance of green. Positive b* values indicate a yellow tendency, whereas the negative range values demonstrate a bluish tone.

It was found that most samples in each group exhibited positive $a *$ and $b *$ changes in hue. These findings are in agreement with another study that used tea as a staining medium, ${ }^{35}$ and have been attributed to certain red and yellow polyphenolic chromogens. ${ }^{36,37}$

According to the results of this study, it was noticed that TempSpan (bis-acryl composite-based material) group recorded the highest color change mean value $(3.68 \pm 0.39 \Delta \mathrm{E})$ followed by Revotek LC (bis-acryl composite-based material) group mean value $(1.56 \pm 0.27 \Delta \mathrm{E})$ while Jet tooth shade (PMMA-based material) group recorded the lowest color change mean value, however, Revotek LC \& Jet tooth shade material groups were not significantly different from each other. These findings may be attributed to the homogeneous composition of the acrylic-based material and the heterogeneous composition of the composite material. The discoloration might be due to both surface adsorption and absorption of the colorants of the oral rinses. Fine colorant particles may have precipitate in the small pits of the bis-acryl composites. The less polar colorants and water-soluble polyphenols in the colorants may have penetrated deeper into the materials, because such colorants are more possibly compatible with the polymer matrix of the composites than with methylmethacrylate. ${ }^{24}$ The results of this study are consistent with a study of Doray et $\mathbf{a l}^{6}$ who stated that MMA provided better color stability. Turgut et $\mathrm{al},{ }^{20}$ also compared the discoloration of provisional restoration materials: one PMMA-based (TemDent Classic $($ ) $)$ and three different bis-acrylic-based (Protemp II ${ }^{\circ}$, Luxatemp ${ }^{\circledR}$ and Fill-In $®$ ) after immersion in different oral rinses. It was concluded that the lowest color change was observed in PMMA-based Temdent in all oral rinses

Additionally, immersion of provisional materials in various staining solutions for up to 1 month indicated that, the MMA materials showed the best color stability and the bis-acryl composite materials were the worst in color stability. ${ }^{11}$ The more color change of TempSpan as a dual curing compositebased material over Revotek LC with light curing mode may be attributed to initial chemical curing with concomitant oxidation of the polymer matrix or unreacted double bonds thus chemical discoloration of the material itself may also have occurred (intrinsic discoloration), ${ }^{6,11}$

At the same time, the composite-based resins is claimed to have the ability to absorb water at a higher rate because of a high diffusion coefficient in 
comparison to MMA-based resins. Such properties may explain the higher color changes observed with composite over MMA- based materials with the use of oral rinses. ${ }^{38}$

It has been stated that MMA-based provisional materials exhibit smoother surfaces after initial polishing in comparison to bis-acryl-based materials. This can explain greater surface roughness \& significant discoloration of bis-acrylic-based provisional materials in the present study. ${ }^{11}$

The color measurements for different provisional materials at baseline and after staining were recorded. Two auto-cure materials, Protemp Garant and Integrity, and one dual-cure material, Luxatemp Solar, were tested against SNAP, a polyethyl methacrylate control. Protemp Garant exhibited a clinically noticeable change in shade after 1 week in staining solution, whereas the other materials did not exhibit a clinically noticeable change, results that are also in agreement with our findings. ${ }^{39}$

In contrast to the result of the present study, color changes associated with continuous exposure to UV light irradiation were studied and compared. ${ }^{12}$ In this study, the bis-acryl composites demonstrated better resistance to discoloration than the PEMA materials.

It also contradicts with another study, ${ }^{40}$ that evaluated the color stability of provisional restorative materials; self-cure tooth moulding material, Protemp 4 and Revotek LC after exposure to different mouth rinses at varying time intervals, Revotek LC was the most color stable provisional crown material for 1 week and after 1-month time interval and this is in agreement with the results of the study conducted. ${ }^{41}$ Significant color change was seen when Revotek LC was tested for 3 months, the result that was similar to the conclusion drawn by Guler et al. ${ }^{9}$

Based on spectrophotometric values obtained in this study, artificial saliva recorded the highest color change mean value $(2.78 \pm 1.2 \Delta \mathrm{E})$ followed by Tantum V solution mean value $(1.72 \pm 0.31 \Delta \mathrm{E})$ then Oracin solution mean value $(1.5 \pm 0.4 \Delta \mathrm{E})$ while CHX staining solution recorded the lowest color change mean value $(1.32 \pm 0.4 \Delta \mathrm{E})$ however, Tantum $\mathrm{V}$, Oracin and effects of Chlorohexidine solution were not significantly different from each other. The highest color change occurred with artificial saliva may be attributed to accumulation of pellicles acting as a matrix for the deposition of stains, which may result in discoloration. It is also consisting mainly of deionized water which is found to play an important role in chemical degradation process such as oxidation and hydrolysis and thereby subsequent change in the optical property of the provisional restorative material.

The discoloration potential of mouth rinses which was confirmed in this study have already been reported in the literature. It was documented that the use of oral rinses for three weeks caused eye perceptible discoloration of natural teeth, which was clinically unacceptable ${ }^{17}$

Oral rinse solutions have various components such as detergents, emulsifiers, organic acids and dyes; which may affect the color of different restorative materials. ${ }^{20}$

Mouthwashes/gels affected color shifting for all resin materials, and changes are exaggerated over time. However, discoloration effects are not perceptible to the human eye. ${ }^{43}$

The staining ability of two mouth rinses namely hexidine and periogard was evaluated. It was indicated that synthetic saliva + hexidine stain more when compared to synthetic saliva + periogard, after 1 week, 1 month and even 3 months. Staining may be caused by destruction mechanism of alcohol containing mouth rinses. ${ }^{40}$

Alcohol containing and alcohol-free mouth rinses affect surface properties of resin restorative material irrespective of alcohol concentrations. ${ }^{44}$ Additionally, chlorhexidine-containing mouth rinses discolored the provisional restorative 
materials more than the synthetic saliva control group..$^{20}$ However, chlorhexidine gluconate, benzydamine hydrochloride and the hybrid solution were not significantly different from each other.

\section{CONCLUSIONS}

1- All the bis-acryl resins and PMMA-based provisional materials showed clinically perceptible color changes after exposure to oral rinses.

2- Among the three provisional restorative materials, TempSpan was found to be significantly the least color stable material and the least stain was taken up by Jet.

3- There were no significant differences between the discoloration effects of Tantum V, Oracin and Chlorohexidine mouth washes on the three tested provisional materials.

\section{CLINICAL RECOMMENDATIONS}

Jet tooth shade followed by Revotek LC provisional materials are more preferred than TempSpan when esthetics is of prime importance.

\section{REFERENCES}

1. Haselton DR, Diaz-Arnold AM, Dawson DV. Effect of storage solution on surface roughness of provisional crowns and fixed partial denture materials. J Prosthodont 2004;13:227-32.

2. Rosenstiel SF, Land M, Fujimoto J. Contemporary fixed prosthodontics. 3rd ed. St Louis: Mosby. 2000;380-416.

3. 3-Sham AS, Chu FC, Chai J, Chow TW. Color stability of provisional prosthodontic materials. J Prosthet Dent 2004;91: 447-452.

4. Scotti R, Mascellani SC, Forniti F. The in vitro colour stability of acrylic resin for provisional restorations. Int $\mathrm{J}$ Prosthodont 1997; 10: 164-168.

5. Galindo D, Soltys JL, Graser GN. Long-term reinforced fixed provisional restorations. J Prosthet Dent 1998; 79:698-701.

6. Doray PG, Li D, Powers JM. Color stability of provisional restorative materials after accelerated aging. J Prosthodont 2001;212-216.
7. Dory PG, Wang X, Powers JM, Burgess J. Accelerated Aging Affects Color Stability of Provisional Restorative Materials. J Prosthodont 1997; 6:183-188.

8. Seghi RR, Gritz MD, Kim J. Colorimetric changes in composites resulting from visible-light-initiated polymerization. Dent Mater 1990; 6:133-137.

9. Guler AU, Yilmaz F, Kulunk T, Guler E, Kurt S. Effects of different drinks on stainability of resin composite provisional restorative materials. J Prosthet Dent 2005; 94:118-124.

10. Crispin BJ and Caputo AA. Color stability of temporary restorative materials. J Prosthet Dent 1979;42:27-33.

11. Yannikakis SA, Zissis AJ, Polyzois GL, Caroni C. Color stability of provisional resin restorative materials. J Prosthet Dent 1998;80:533-9.

12. Sham AS, Chu FC, Chai J, Chow TW. Color stability of provisional prosthodontic materials. J Prosthet Dent 2004;91:447-52.

13. Scotti R, Mascellani SC, Forniti F. The in vitro color stability of acrylic resins for provisional restorations. Int $\mathrm{J}$ Prosthodont 1997;10:164-168.

14. Koumjian JH, Firtell DN, Nimmo A. Color stability of provisional materials in vivo. J Prosthet Dent 1991;65: $740-742$

15. Luce MS, Campbell CE. Stain potential of four microfilled composites. J Prosthet Dent 1988;60:151-154.

16. Stober T, Gilde H, Lenz P. Color stability of highly filled composite resin. Dent Mater 2001;17:87-94.

17. Bagis B, Baltacioglu E, Özcan M, Ustaomer S. Evaluation of chlorhexidine gluconate mouth rinse-induced staining using a digital colorimeter: an in vivo study. Quintessence Int. 2011; 42:213-23.

18. Sorensen JA, Doherty FM, Newman MG, Flemmig TF. Gingival enhancement in fixed prosthodontics. Part I: Clinical findings. J Prosthet Dent 1991; 65:100-7.

19. Yumiko HY, Watanabe E, Tadokoro K, Inoue T, Miyazaki M, Franklin R. Effects of ammonium hexafluorosilicate application on demineralized enamel and dentin of primary teeth. Oral Sci. 2012; 54:267-272.

20. Turgut S, Bagis B, Ayaz E, Ulusoy K, Altintas S, Korkmaz F. Discoloration of Provisional Restorations after Oral Rinses. Int. J. Med. Sci. 2013; 10:1503-1509.

21. Burns DR, Beck DA, Nelson SK. A review of selected dental literature on contemporary provisional fixed prosthodontic 
treatment: Report of the Committee on Research in Fixed Prosthodontics of the Academy of Fixed Prosthodontics. J Prosthet Dent 2003;90:474-97.

22. Doray PG, Eldiwany MS, Powers JM. Effect of resin surface sealers on improvement of stain resistance for a composite provisional material. J Esthet Restor Dent 2003; 15:244-250.

23. Guler ${ }^{\mathrm{a}} \mathrm{AU}$, Kurt ST, Kulunk T. Effects of various finishing procedures on the staining of provisional restorative materials. J Prosthet Dent 2005;93:453-8.

24. Haselton DR, Diaz-Arnold AM, Dawson DV. Color stability of provisional crown and fixed partial denture resins. J Prosthet Dent 2005;93:70-5.

25. Diaz-Arnold AM, Dunne JT, Jones AH: Microhardness of provisional fixed prosthodontic materials. J Prosthet Dent 1999; 82:525-528.

26. Ireland MF, Dixon DL, Breeding LC, et al: In vitro mechanical property comparison of four resins used for fabrication of provisional fixed restorations. J Prosthet Dent 1998;80:158-162.

27. Young HM, Smith CT, Morton D. Comparative in vitro evaluation of two provisional restorative materials. J Prosthet Dent 2001;85:129-132.

28. Bagheri R, Burrow MF, Tyas M. Influence of foodsimulating solutions and surface finish on susceptibility to staining of aesthetic restorative materials. J Dent 2005;33:389-398.

29. Um CM, Ruyter IE. Staining of resin-based veneering materials with coffee and tea. Quint Int 1991;22:377-86.

30. Keyf F, Anil H. The effect of margin design on the marginal adaptation of temporary crowns. J Oral Rehabil 1994;21:367-71.

31. Wyszecki G, Fielder GH. Color difference matches. J Opt Soc Am A 1971; 61:1501-1513.

32. Billmeyer FW, Saltzman M. Observer metamerism. Color Res Appl 1980;5:72.
33. Brewer DJ, Wee A, Seghi R. Advances in color matching. Dent Clin N Am 2004;48:341-358.

34. O'Brien WJ: Dental Materials and Their Selection (ed 3). Chicago, IL, Quintessence, 2002, p. 28.

35. Sulieman M, Addy M, Rees JS: Development and evaluation of a method in vitro to study the effectiveness of tooth bleaching. J Dent 2003;31:415-422.

36. Harler CR: Tea Manufacture. London, Oxford United Press, 1963, pp. 13-22.

37. Pearson D: The Chemical Analysis of Foods (ed 7). London, Churchill Livingston, 1976.

38. Bernardi F, Pincelli MR, Carloni S, Gatto MR, Montebugnoli L. Chlorhexidine with an antidiscoloration system. A comparative study. Int J Dent Hyg. 2004; 2:122-126.

39. Givens EJ, Jr., Neiva G, Yaman P, \& Dennison JB. Marginal Adaptation and Color Stability of Four Provisional Materials. Int J Prosthodont 2008;17: 97-101 .

40. Prasad DK, Alva H, Shetty M: Evaluation of Colour Stability of Provisional Restorative Materials Exposed to Different Mouth Rinses at Varying Time Intervals: An In Vitro Study. J Indian Prosthodont Soc 2014; 14:85-92.

41. Gupta G and Gupta T. Evaluation of the effect of various beverages and food material on the color stability of provisional materials: an in vitro study. J Conserv Dent 2011;14:287-292.

42. Guler AU, Yilmaz F, Kulunk T, Guler E, Kurt S. Effects of different drinks on stainability of resin composite provisional restorative materials. J Prosthet Dent 2005;94:118-124.

43. Al-Samadani KH: The Effect of Preventive Agents (Mouthwashes/Gels) on the Color Stability of Dental ResinBased Composite Materials. Dent. J. ;2017: 5(2), 18.

44. Gurgan S, Onen A, Koprulu H. In vitro effects of alcohol containing and alcohol-free mouth rinses on microhardness of some restorative materials. J Oral Rehabil1997; 24: 244-246. 